\title{
Effect of heat treatment on stainless steel orthodontic wires
}

\begin{abstract}
Osmar Aparecido Cuoghi(a) Geraldo Francisco Kasbergen ${ }^{(b)}$ Paulo Henrique dos Santos ${ }^{(c)}$ Marcos Rogério de Mendonça(a) Pedro Marcelo Tondelli ${ }^{(\mathrm{d})}$
\end{abstract}

\footnotetext{
(a) Department of Pediatric and Community Dentistry, Faculdade de Odontologia de Araçatuba, Unesp - Univ Estadual Paulista, Araçatuba, SP, Brazil.

(b) Department of Pediatric Dentistry, Faculdade de Odontologia, Universidade de Itaúna, Itaúna, MG, Brazil.

(c) Department of Dental Materials and Prosthodontics, Faculdade de Odontologia de Araçatuba, Unesp - Univ Estadual Paulista, Araçatuba, SP, Brazil.

(d) Faculdade de Odontologia de Araçatuba, Unesp - Univ Estadual Paulista, Araçatuba, SP, Brazil.
}

\begin{abstract}
This study evaluated the effect of heat treatment on $\mathrm{CrNi}$ stainless steel orthodontic archwires. Half of forty archwires of each thickness - 0.014" (0.35 mm), 0.016" (0.40 mm), 0.018” (0.45 mm) and $0.020 "(0.50 \mathrm{~mm})$ (totalling 160 archwires) - were subjected to heat treatment while the remainder were not. All of the archwires had their individual thickness measured in the anterior and posterior regions using AutoCad 2000 software before and after compressive and tensile strength testing. The data was statistically analysed utilising multivariance ANOVA at a 5\% significance level. All archwires without heat treatment that were subjected to tensile strength testing presented with anterior opening, which was more accentuated in the 0.020 " archwires. In the posterior region, the opening produced by the tensile force was more accentuated in the archwires without heat treatment. There was greater stability in the thermally treated archwires, especially those subjected to tensile strength testing, which indicates that the heat treatment of orthodontic archwires establishes a favourable and indispensable condition to preserve the intercanine width.
\end{abstract}

Descriptors: Orthodontic Wires; Stainless Steel; Tooth Movement.

\section{Introduction}

The effectiveness of orthodontic movement involves the adequate interaction of factors related to the patient, mechanics, teeth and periodontal supporting structures. The outcome of treatment is particularly dependent upon the action of the orthodontic wires, according to their structural and mechanical characteristics. ${ }^{1}$

In the traditional sequence of replacing stainless steel wires during the levelling and alignment phases, the progressive transition from thinner to thicker wires alter the amount of force released. ${ }^{2}$ When the wire receives a tensile force before reaching its limit of proportionality, it will respond by returning to its original form and will therefore be in its elastic phase. After passing the elastic limit, the wire will reach the plastic phase when it changes its form, yet without returning to its original shape. If an exaggerated force is applied, a permanent deflection occurs and the wire no longer returns to its original form. This occurs because the deflection surpasses the elastic limit of the wire. ${ }^{3,4}$

When an orthodontic wire is deformed, several internal tensions occur, which means that its atoms are spatially dislocated and the interatomic forces become unbalanced. This condition of instability is due to
Received for publication on Sep 16, 2010 Accepted for publication on Dec 07, 2010

\author{
Corresponding autho \\ Disciplina de Ortodontia - Faculdade de \\ Rua José Bonifácio, 1193 \\ CEP: 16015-050 \\ E-mail: tondelli.ortodontia@hotmail.com
}


the fact that some atoms get too close to each other while others become too distant. The atoms tend to return to their original position by diffusion with time and a consequence of this process, called stress releasing, is wire distortion. ${ }^{3}$ In order to avoid this phenomenon, after bending, the stainless steel wire is heated to a temperature of $850^{\circ} \mathrm{F}$ for 3 minutes until a reddish-brown colour is obtained. ${ }^{5}$ This procedure is known as "heat treatment" and recovers the normal aspect of the metal microstructure. ${ }^{4-6}$ Another method of performing this treatment is passing the bent wire repeatedly through the flame of an alcohol lamp until a reddish-brown colour is observed in its entire extension. However, this procedure does not follow a technical standardisation.

Clinically, when the orthodontist makes loops, bends or establishes a new arch form, the wire reaches a high internal pressure and should be thermally treated in order to release these tensions. ${ }^{7}$ Only conflicting and insufficient information is available regarding the alterations induced by the heat treatment, which makes this treatment a controversial choice in orthodontics. These factors, allied with the existence of only a small number of reported studies regarding this subject, were the rationale for the present study. To the best of our knowledge, all available studies refer to laboratorial investigations and do not address this procedure under clini- cal conditions. It is common in orthodontic practice to use an alcohol lamp or a welding machine with a specific device for heat treatment. However, neither of these procedures follows rigorous time and temperature standards. Therefore, since these procedures are routinely performed by the majority of orthodontists, it is important to investigate their effect on the physical properties of stainless steel wires. The purpose of this study was to evaluate the effect of heat treatment on stainless steel orthodontic archwires of different thicknesses when subjected to compressive and tensile strength forces.

\section{Material and methods}

One hundred and sixty $150-\mathrm{mm}$-long round chromium-nickel stainless steel orthodontic wires (Morelli, Sorocaba，SP, Brazil) with thicknesses of $0.014 ”(0.35 \mathrm{~mm}), 0.016 "(0.40 \mathrm{~mm}), 0.018$ ” $(0.45 \mathrm{~mm})$ and $0.020 "(0.50 \mathrm{~mm})$ were used. Forty archwires of each thickness were made by a single operator using, as reference, the OrthoForm III diagram (3M/Unitek, St. Paul, MN, USA) (Figure 1), and were randomly chosen to receive or not heat treatment ( $\mathrm{n}=20$ for each condition). Half of the thermally and non-thermally treated archwires were subjected to a compressive strength test and half were subjected to a tensile strength test $(\mathrm{n}=10$ for each condition). The forces were applied to both
Figure 1 - Archwire with demarcations positioned onto the OrtoForm III Diagram - 3M/Unitek, St. Paul, MN, USA.

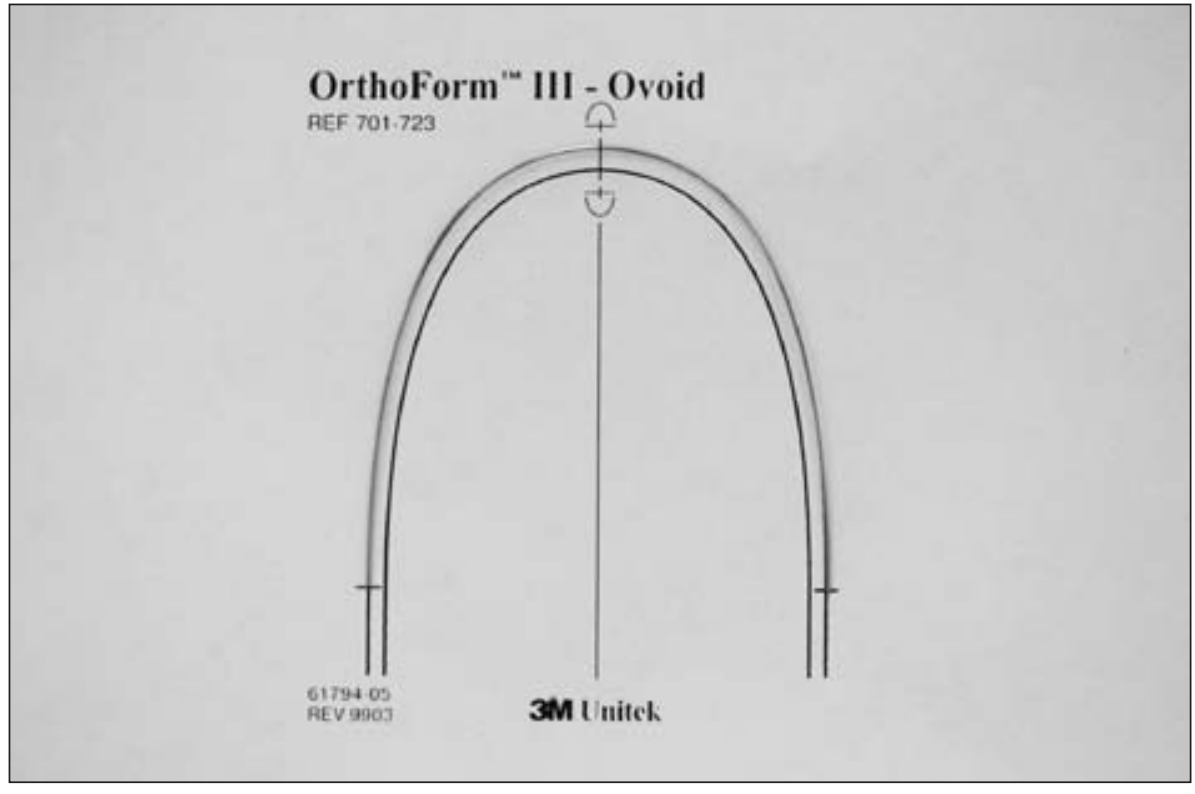


archwire ends.

Transverse demarcations were plotted in the anterior and posterior regions of the archwires using a diagram traced on an A4 sheet of graph paper in order to analyse the occurrence of transverse alterations in these regions. First, a horizontal line, perpendicular to a vertical midline, was traced at the ends of the archwire to serve as a reference limit (A). Then, a new horizontal line (B) was traced parallel to the previous line and $20 \mathrm{~mm}$ above it, thus creating a reference for demarcation of two bilateral points in the posterior region of the archwire and establishing the intermolar width. The transverse distance demarcated at the anterior region of the archwires $(\mathrm{C})$ corresponded to a mean intercanine width of $35 \mathrm{~mm}$, which has been established by van der Linden. ${ }^{8}$ A standard diagram was thus obtained for demarcation of the four points corresponding to the intercanine and intermolar widths (Figure 2).

Each archwire was individually superposed onto the diagram in order to bilaterally demarcate the points corresponding to the intercanine and intermolar widths for subsequent measurement (Figure 2). Next, heat treatment was performed on 20 archwires of each thickness using a welding machine (model SMP 3000; Kernit Indústria Mecatrônica Ltda., Indaiatuba, SP, Brazil; $500 \mathrm{~W}$ power, 4.0/2.0 A current, $50 / 60 \mathrm{~Hz}$ frequency) with a special device
(Figure 3). The machine was set at a power setting of 3 and an electric current time of 8 seconds was set for all thermally treated archwires. The remaining 20 archwires of each thickness did not receive heat treatment.

All 160 archwires were scanned with a desktop scanner using 2400 dpi acquired resolution (HP PSC 1510 All-in-One, Hewlett Packard, Sorocaba, SP, Brazil) and measurements were carried out in the anterior and posterior regions before and after the compressive and tensile strength tests, using AutoCad 2000 software (Autodesk Inc., San Rafael, CA, USA). The mechanical tests were performed in a universal testing machine (model DL3000; EMIC, São José dos Pinhais, PR, Brazil) with a $200 \mathrm{~N}$ load cell and a crosshead speed of $2 \mathrm{~cm} / \mathrm{min}$. The compressive and tensile strength tests were standardised at a distance of $40 \mathrm{~mm}$ due to the limitations of the testing machine during application of the compression force. The data was statistically analysed by one-way multivariance ANOVA at a 5\% significance level.

\section{Results 0.014" archwires}

A statistically significant difference was only found between the transverse distances in the posterior region, obtained before and after tensile

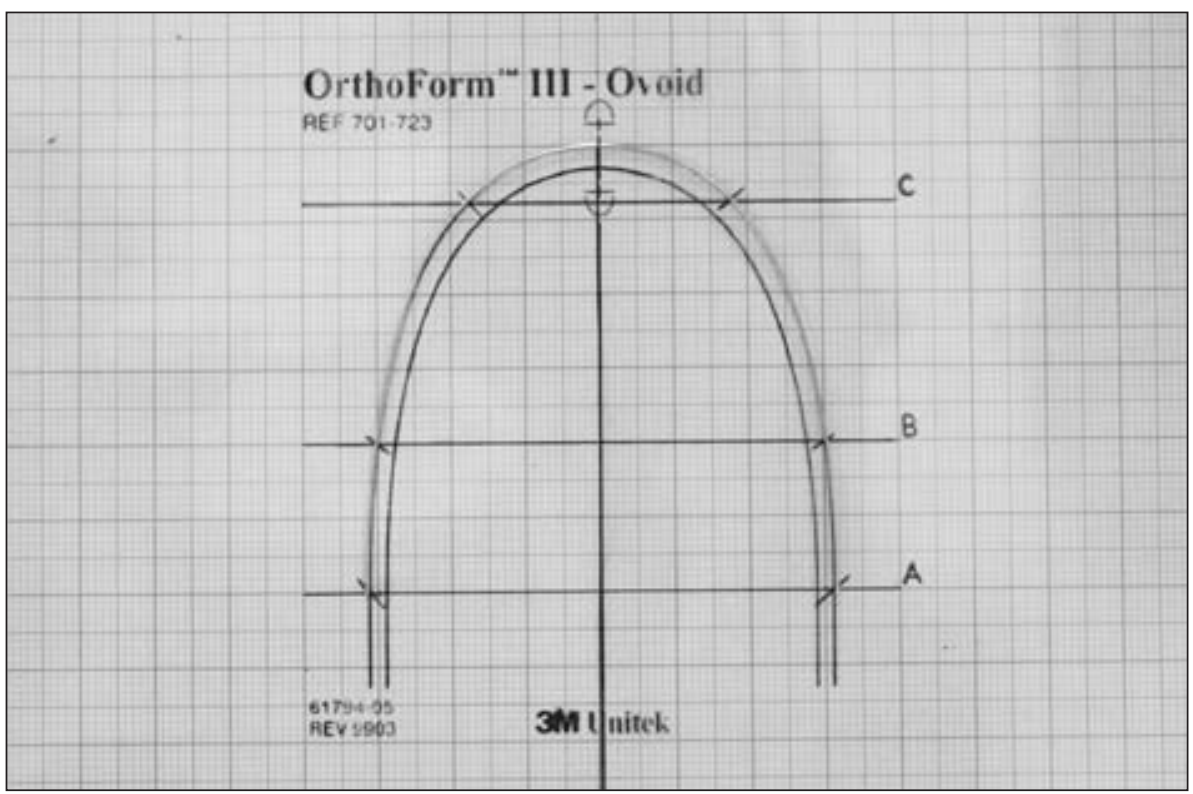

Figure 2 - Archwire superposed on the diagram with demarcation of the points in the anterior and posterior regions. 
Figure 3 - Welding machine with the special device for heat treatment.

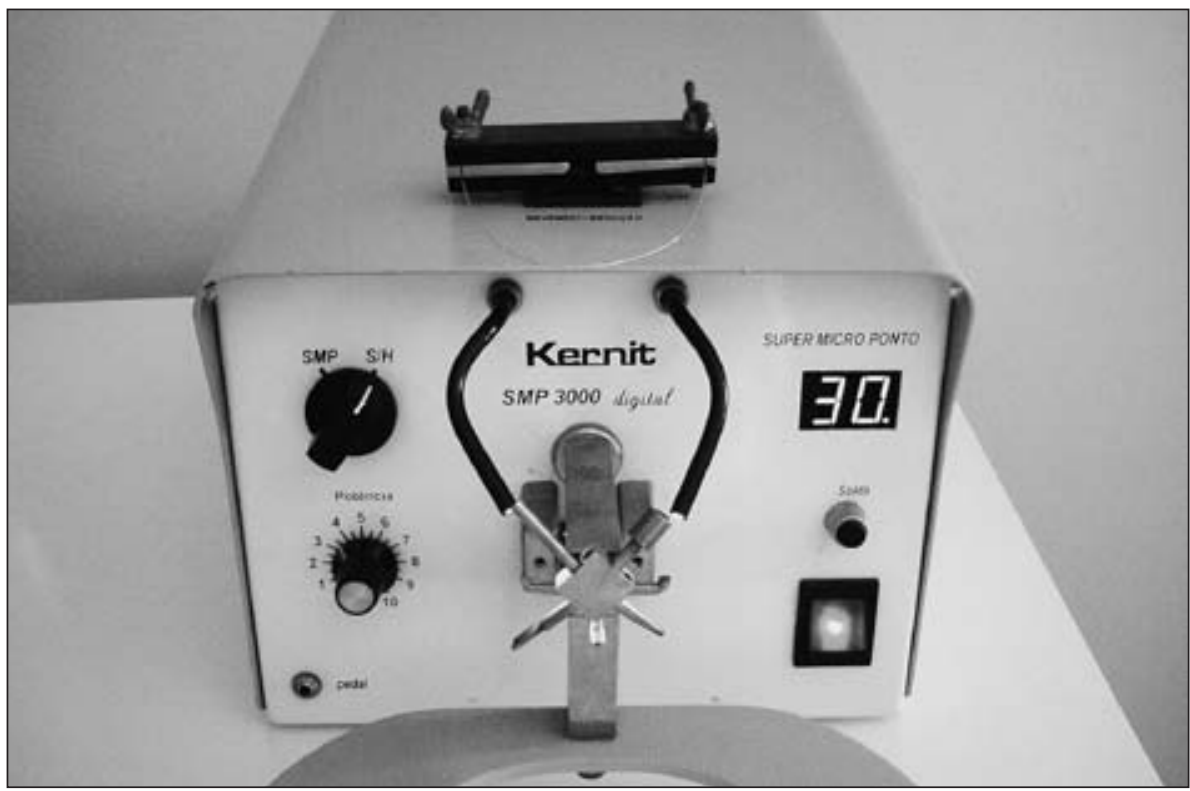

strength testing for both the heat-treated archwires (hereafter referred to as "HT archwires") and the archwires without heat treatment (hereafter referred to as "WHT archwires") ( $\mathrm{p}=0.0241$ and $\mathrm{p}=0.0000063$, respectively) (Table 1 and 2 ).

\section{$0.016^{\prime \prime}$ archwires}

In the posterior region, a significant difference was identified between the transverse distances obtained before and after the compressive strength test for the WHT archwires ( $p=0.0007)$. A statistically significant difference was found after the tensile strength test for both the HT and WHT archwires ( $\mathrm{p}=0.0124$ and $\mathrm{p}=0.0000019$, respectively) (Table 1 and 2).

\subsection{8 " archwires}

In the anterior region, a significant difference was established between the transverse distances obtained before and after the compressive strength test for the HT archwires ( $\mathrm{p}=0.0003)$. In the posterior region, there was a significant difference before and after the tensile strength test for both HT and WHT archwires ( $\mathrm{p}=0.0475$ and $\mathrm{p}=0.0000037$, respectively) (Table 1 and 2).

\subsection{0 " archwires}

A significant difference was found in the ante- rior region before and after the tensile strength test for both HT and WHT archwires ( $\mathrm{p}=0.0404$ and $\mathrm{p}=0.0025$, respectively).

In the posterior region, a statistically significant difference was identified before and after the compressive strength test for the WHT archwires $(\mathrm{p}=0.0187)$, and in the same way before and after the tensile strength test for both the HT and WHT archwires $(\mathrm{p}=0.00003$ and $\mathrm{p}=0.0000001$, respectively (Table 1 and 2 ).

\section{Discussion}

One of the major requisites of orthodontic mechanics is the maintenance of dental arch dimensions using wires during dental movement. At completion of the tooth levelling/alignment phase, which is guided by the analysis of the orthodontic diagram and the use of different wire dimensions, a satisfactory intra- and inter-arch relationship must be observed. ${ }^{9}$ Claro et al. ${ }^{10}$ suggested that the increase in arch perimeter is approximately given by the addition of 0.54 times the intercanine expansion, which is useful in solving crowding cases, but Rudge ${ }^{11}$ and de la Cruz et al. ${ }^{12}$ have advised that arch shape is the best guidance for future stability. The use of pre-adjusted orthodontic accessories facilitates and simplifies arch contouring, but does not eliminate the need for individualising orthodontic archwires. ${ }^{13}$ 
Table 1 - Mean values and standard deviations (SD) in $\mathrm{mm}$ of the transverse dimensions in the anterior and posterior region of the $0.014^{\prime \prime}, 0.016^{\prime \prime}, 0.018^{\prime \prime}$ and $0.020^{\prime \prime}$ archwires with and without heat treatment before and after the compressive strength test.

\begin{tabular}{|c|c|c|c|c|c|}
\hline \multirow{2}{*}{ Archwire Thickness } & \multirow{2}{*}{ Heat Treatment } & \multicolumn{2}{|c|}{ Anterior Region } & \multicolumn{2}{|c|}{ Posterior Region } \\
\hline & & Before Testing $(n=10)$ & After Testing $(n=10)$ & Before Testing $(n=10)$ & After Testing $(\mathrm{n}=10)$ \\
\hline \multirow{2}{*}{$0.014^{\prime \prime}(n=40)$} & No $(n=20)$ & $35.00(0.36)^{a}$ & $34.84(0.34)^{a}$ & $59.81(0.27)^{a}$ & $59.61(0.34)^{a}$ \\
\hline & Yes $(n=20)$ & $34.80(0.37)^{a}$ & $34.77(0.41)^{a}$ & $61.57(0.91)^{a}$ & $61.33(1.02)^{a}$ \\
\hline \multirow{2}{*}{$0.016^{\prime \prime}(n=40)$} & No $(n=20)$ & $35.14(0.50)^{a}$ & $34.96(0.49)^{a}$ & $59.87(0.18)^{a}$ & $59.15(0.51)^{b}$ \\
\hline & Yes $(n=20)$ & $35.09(0.43)^{a}$ & $35.10(0.42)^{\mathrm{a}}$ & $59.85(0.39)^{a}$ & $59.65(0.36)^{\circ}$ \\
\hline \multirow{2}{*}{$0.018^{\prime \prime}(n=40)$} & No $(n=20)$ & $35.33(0.53)^{a}$ & $35.26(0.56)^{a}$ & $59.64(0.13)^{a}$ & $59.47(0.31)^{a}$ \\
\hline & Yes $(n=20)$ & $35.38(0.20)^{a}$ & $34.96(0.21)^{b}$ & $62.07(1.14)^{a}$ & $61.70(1.21)^{\circ}$ \\
\hline \multirow{2}{*}{$0.020^{\prime \prime}(n=40)$} & No $(n=20)$ & $34.89(0.21)^{a}$ & $34.81(0.25)^{a}$ & $59.91(0.24)^{a}$ & $59.52(0.41)^{b}$ \\
\hline & Yes $(n=20)$ & $34.94(0.50)^{a}$ & $35.03(0.48)^{a}$ & $62.81(0.69)^{a}$ & $62.85(0.69)^{a}$ \\
\hline
\end{tabular}

Different letters indicate statistically significant difference $(p<0.05)$.

Table 2 - Mean values and standard deviations (SD) in $\mathrm{mm}$ of the transverse dimensions in the anterior and posterior region of the $0.014^{\prime \prime}, 0.016^{\prime \prime}, 0.018^{\prime \prime}$ and $0.020^{\prime \prime}$ archwires with and without heat treatment before and after the tensile strength test.

\begin{tabular}{|c|c|c|c|c|c|}
\hline \multirow{2}{*}{ Archwire Thickness } & \multirow{2}{*}{ Heat Treatment } & \multicolumn{2}{|c|}{ Anterior Region } & \multicolumn{2}{|c|}{ Posterior Region } \\
\hline & & Before Testing $(n=10)$ & After Testing $(n=10)$ & Before Testing $(n=10)$ & After Testing $(n=10)$ \\
\hline \multirow{2}{*}{$0.014^{\prime \prime}(n=40)$} & No $(n=20)$ & $35.02(0.37)^{a}$ & $35.21(0.37)^{a}$ & $59.63(0.22)^{a}$ & $61.88(0.83)^{\mathrm{b}}$ \\
\hline & Yes $(n=20)$ & $34.74(0.49)^{a}$ & $34.94(0.57)^{a}$ & $61.38(0.59)^{a}$ & $62.09(0.70)^{b}$ \\
\hline \multirow{2}{*}{$0.016^{\prime \prime}(n=40)$} & No $(n=20)$ & $34.87(0.53)^{a}$ & $35.31(0.60)^{a}$ & $59.80(0.34)^{a}$ & $62.75(0.87)^{\mathrm{b}}$ \\
\hline & Yes $(n=20)$ & $35.04(0.45)^{a}$ & $35.17(0.45)^{a}$ & $60.36(0.60)^{a}$ & $61.29(0.88)^{b}$ \\
\hline \multirow{2}{*}{$0.018^{\prime \prime}(n=40)$} & No $(n=20)$ & $35.03(0.48)^{a}$ & $35.49(0.54)^{a}$ & $59.83(0.24)^{a}$ & $62.72(0.99)^{\mathrm{b}}$ \\
\hline & Yes $(n=20)$ & $35.41(0.52)^{a}$ & $35.45(0.45)^{a}$ & $62.75(0.98)^{a}$ & $63.70(1.04)^{b}$ \\
\hline \multirow{2}{*}{$0.020^{\prime \prime}(n=40)$} & No $(n=20)$ & $34.91(0.41)^{a}$ & $35.69(0.56)^{b}$ & $59.66(0.34)^{a}$ & $65.06(0.92)^{b}$ \\
\hline & Yes $(n=20)$ & $35.10(0.28)^{a}$ & $35.46(0.44)^{\mathrm{b}}$ & $63.10(0.53)^{a}$ & $64.85(0.68)^{b}$ \\
\hline
\end{tabular}

Different letters indicate statistically significant difference $(p<0.05)$.

In the tooth leveling/alignment phase, when stiffer and thicker wires are required, stainless steel archwires are frequently used according to pre-established individual diagrams. During contouring, stainless steel wires reach a high level of internal tension and require heat treatment for release of this tension. ${ }^{3,7}$ The increased elastic strength is the most significant effect of heat treatment. An elastically stronger working appliance is more likely to return to its original shape, without suffering any permanent deflection. ${ }^{14}$

In the present study, all WHT archwires subjected to compressive strength testing presented with little decrease in the width in the anterior region before and after the mechanical test, though without statistical significance.

All WHT archwires subjected to tensile strength testing presented with a tendency towards progressive and more accentuated transverse alterations in the anterior region, according to the wire thickness. In the 0.020" WHT archwires, the intercanine width increased significantly from 34.91 to $35.69 \mathrm{~mm}$, more than HT archwires which increased from 35.10 to $35.46 \mathrm{~mm}$ (Table 2). These findings show that, when no heat treatment is performed, as the wire thickness increases, then the forces that cause wire expansion may modify the intercanine width.

In the anterior region, which is commonly referred to in literature as responsible for most re- 
lapses after orthodontic treatment, ${ }^{15}$ the WHT archwires had a greater tendency to open after the tensile strength test. These results establish an important clinical safety procedure: to perform the HT on stainless steel orthodontic wires, thus preserving the intercanine distance during tooth leveling and alignment. Little ${ }^{15}$ and Vanarsdall Junior ${ }^{16}$ demonstrated that the main reason for the recurrence of antero-inferior tooth crowding is the increase in the intercanine distance during orthodontic treatment.

In the posterior region, all WHT archwires presented with a decrease in the transverse distance to compressive strength, which was significant for the 0.016 " and 0.020" archwires.

All WHT archwires presented with a significantly greater opening after the tensile strength test. The 0.020 " archwires exhibited an increase of approximately $6 \mathrm{~mm}$, while the other wire thicknesses presented with openings between 2 and $3 \mathrm{~mm}$ (Table 2). These results revealed a "difficulty" in preserving the intermolar distance when the orthodontic mechanics promotes an opening force in WHT archwires and this was shown most evidently in the expansion of the thicker archwire (0.020"). The effect of heat treatment is evident on HT archwires, which indicated an increase of $1.75 \mathrm{~mm}$ (0.020") and the other wire thicknesses presented with openings of approximately $1 \mathrm{~mm}$.

For the tensile strength testing performed in the anterior region of the HT archwires, slight openings were observed for the 0.014 ", 0.016 " and 0.018 " archwires. However, in the 0.020 " archwires, the transverse distance increased by $0.36 \mathrm{~mm}$, that is, they presented with a greater tendency than the other wire thicknesses to an increase of the transverse distance in the anterior region, when subjected to tensile strength testing.

The results of this study demonstrated that the

\section{References}

1. Burstone CJ. Variable-modulus orthodontics. Am J Orthod. $1981 \mathrm{Jul} ; 80(1): 1-16$.

2. Walters NE. A rationale for the selection of orthodontic wires. Eur J Orthod. 1992 Jun;14(3):240-5.
HT performed in daily clinical practice establishes a favourable and determinant condition to preserve intercanine width. Several authors have expressed their concern with the maintenance of the intercanine width in the post-orthodontic treatment period. ${ }^{9,17,18}$

The archwires subjected to the HT and compressive strength testing presented with a tendency to reduce the posterior transverse distance though in smaller proportions, since no significant differences were observed in any statistical comparisons. The results demonstrate a greater shape stability of the HT archwires when subjected to compressive strength testing.

During the compressive and tensile strength tests, the HT archwires demonstrated a smaller tendency to transverse changes in the anterior and posterior regions, when compared to WHT archwires. Therefore further studies should be performed.

\section{Conclusions}

Based on the employed methodology and the obtained results, the following conclusions may be drawn:

1. The archwires with and without heat treatment subjected to compressive strength testing presented with minor transverse changes in the anterior and posterior regions.

2. All archwires without heat treatment subjected to tensile strength testing presented with significant openings in the posterior region. In the anterior region, openings also occurred in all archwires, but they were more accentuated in the 0.020 " archwires.

3. The archwires which experienced heat treatment were more resistant to the transverse changes in both anterior and posterior regions when subjected to tension.

3. Gjerdet NR, Hero H. Metal release from heat-treated orthodontic archwires. Acta Odontol Scand. 1987 Dec;45(6):40914.

4. Kusy RP. A review of contemporary archwires: their properties and characteristics. Angle Orthod. 1997 Jun;67(3):197-207. 
5. Funk AC. The Heat-treatment of the stainless-steel. Angle Orthod. 1951 Jul;21(3):129-38.

6. Kohl RW. Metallurgy in orthodontics. Angle Orthod. 1964 Jan;34(1):37-62.

7. Oh KT, Kim KN. Ion release and cytotoxicity of stainless steel wires. Eur J Orthod. 2005 Dec;27(6):533-40.

8. van der Linden FPGM. Development of the dentition. $1^{\text {st }}$ ed. Chicago: Quintessence; 1983. 213 p.

9. Lee RT. Arch width and form: a review. Am J Orthod Dentofacial Orthop. 1999 Mar;115(3):305-13.

10. Claro CAA, Abrão J, Reis SAB, Fantini SM. Correlation between transverse expansion and increase in the upper arch perimeter after rapid maxillary expansion. Braz Oral Res. 2006 Jan-Mar;20(1):76-81.

11. Rudge SJ. Dental arch analysis: arch form. A review of the literature. Eur J Orthod. 1981 Oct-Dec;3(4):279-84.

12. de la Cruz A, Sampson P, Little RM, Årtun J, Shapiro PA. Long-term changes in arch form after orthodontic treat- ment and retention. Am J Orthod Dentofacial Orthop. 1995 May;107(5):518-30.

13. McLaughlin RP, Bennett JC, Trevisi HJ. Systemized orthodontic treatment mechanics. $1^{\text {st }}$ ed. Edinburgh: Mosby; 2001. 324 p.

14. Backofen WA, Gales GF. The low temperature heat-treatment of stainless steel for orthodontics. Angle Orthod. 1951 Apr;21(2):117-24.

15. Little RM. Stability and relapse of mandibular anterior alignment: University of Washington studies. Semin Orthod. 1999 Sep;5(3):191-204.

16. Vanarsdall Junior RL. Transverse dimension and long-term stability. Semin Orthod. 1999 Sep;5(3):171-80.

17. Baluta J, Lavelle CLB. An analysis of dental arch form. Eur J Orthod. 1987 May;9(2):165-71.

18. Bishara SE, Chadra JM, Potter RB. Stability of intercanine width, overbite and overjet correction. Am J Orthod. 1973 Jun;63(6):588-95. 